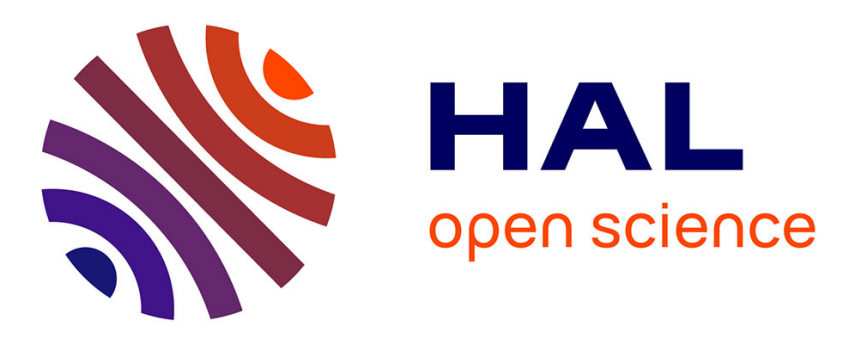

\title{
Nuclear liquid-gas phase transition and supernovae evolution
}

\author{
J. Margueron, J. Navarro, P. Blottiau
}

\section{To cite this version:}

J. Margueron, J. Navarro, P. Blottiau. Nuclear liquid-gas phase transition and supernovae evolution. Physical Review C, 2004, 70, pp.028801. 10.1103/PhysRevC.70.028801 . hal-00001067v2

\section{HAL Id: hal-00001067 \\ https://hal.science/hal-00001067v2}

Submitted on 11 Mar 2004

HAL is a multi-disciplinary open access archive for the deposit and dissemination of scientific research documents, whether they are published or not. The documents may come from teaching and research institutions in France or abroad, or from public or private research centers.
L'archive ouverte pluridisciplinaire $\mathbf{H A L}$, est destinée au dépôt et à la diffusion de documents scientifiques de niveau recherche, publiés ou non, émanant des établissements d'enseignement et de recherche français ou étrangers, des laboratoires publics ou privés. 


\title{
Nuclear liquid-gas phase transition and supernovae evolution
}

\author{
Jérôme Margueron ${ }^{\mathrm{a}, *, 1}$ Jesús Navarro ${ }^{\mathrm{b}}$ Patrick Blottiau ${ }^{\mathrm{c}}$

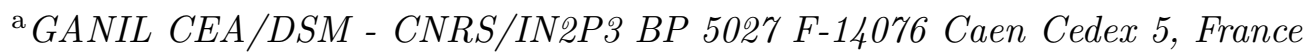 \\ ${ }^{\mathrm{b}}$ IFIC (CSIC - Universidad de Valencia) Apdo. 22085, E-46.071-Valencia, Spain \\ ${ }^{\mathrm{c}}$ CEA/DIF DPTA BP12 F-91680 Bruyères-le-Châtel Cedex, France
}

\begin{abstract}
It is shown that the large density fluctuations appearing at the onset of the first order nuclear liquid-gas phase transition can play an important role in the supernovae evolution. Due to these fluctuations, the neutrino gas may be trapped inside a thin layer of matter near the proto-neutron star surface. The resulting increase of pressure may induce strong particle ejection a few hundred milliseconds after the bounce of the collapse, contributing to the revival of the shock wave. The HartreeFock+RPA scheme, with a finite-range nucleon-nucleon effective interaction, is employed to estimate the effects of the neutrino trapping due to the strong density fluctuations, and to discuss qualitatively the consequences of the suggested new scenario.
\end{abstract}

Key words: neutrinos, supernovae, phase transition, nuclear matter PACS: 97.60.Bw, 26.50.+x, 25.30.Pt, 21.60.Jz

The first simulations of Colgate and White [1] and Arnett [2] have settled the general scenario of explosive supernovae, which schematically goes as follows. Stars with more than about ten times the Sun mass develop central iron cores which eventually become unstable and collapse to neutron stars, due to electron capture and photodisintegration onto iron-group nuclei. The interior of neutron stars is denser than nuclear matter and initially extremely hot. Above nuclear density, the equation of state becomes stiff enough to produce a bounce of the core, and a shock wave is formed, moving to the infalling outer core

\footnotetext{
* Corresponding author.

Email address: margueron@tphys.physik.uni-tuebingen.de (Jérôme Margueron).

1 Present address: Institut für Theoretische Physik, Universität Tübingen, D-72076 Tübingen, Germany
} 
and enveloppe. Particle reactions at such conditions create a huge number of neutrinos, which eventually escape from the dense neutron star, transferring energy to the matter in the still infalling outer layers of the star. This neutrino heating is believed to cause the violent disruption of the star in a supernova explosion. However, from the very beginning it was found that the resulting shock wave is not energetic enough. Recently, an enormous effort has been done to describe the neutrino production and interactions in great detail [3], including the effects of stellar rotation and of violent anisotropic plasma motions. Convective processes in the supernova core had been recognized to accelerate the energy transport inside the neutron star and to enhance the deposition of energy by neutrinos in the outer stellar layers, thus supporting the explosion of the star (the "delayed" mechanism). Along some decades, numerical simulations were performed, starting from the previous $1 \mathrm{D}$ codes $[4,5]$ and the flux-limited neutrino transport scheme [6] to reach the multi-dimensional calculations, showing the importance of convection and Rayleigh-Taylor instabilities, and the most refined neutrino transport methods $[7,8]$. Nevertheless, the outcome of these worldwide most elaborate supernova simulations is disappointing, as no explosions could be obtained. This negative result shatters the widely accepted view of how the explosion starts.

In this letter we point out that large density fluctuations associated to the first order nuclear liquid-gas phase transition, can play an important role in the neutrino trapping. Indeed, the scattering of neutrinos is hugely increased at the onset of strong density fluctuations, so that neutrinos should be trapped inside a thin layer of the proto-neutron star (PNS) for densities in the range between about $0.1 \rho_{0}$ and $0.6 \rho_{0}$, where $\rho_{0}$ is the nuclear matter density at saturation. The resulting increase in the neutrino pressure may induce strong particle ejection a few hundred milliseconds after the bounce of the collapse, contributing to the revival of the shock wave.

The neutrino-nucleon cross section is obtained from a phase space integration of the nuclear response function. In the non-relativistic limit, the neutrino mean-free path $\lambda$ is given by the well-known expression [9]

$$
\frac{1}{\lambda\left(\mathrm{k}_{\nu}, T\right)}=\frac{G_{F}^{2}}{16 \pi^{2}} \int d \mathbf{k}_{3}\left(c_{V}^{2}(1+\chi) \mathcal{S}^{(0)}(q, T)+c_{A}^{2}(3-\chi) \mathcal{S}^{(1)}(q, T)\right)
$$

where $k_{\nu}$ is the neutrino energy-momentum, $T$ is the temperature, $G_{F}$ is the Fermi constant, $c_{V}\left(c_{A}\right)$ the vector (axial) coupling constant, $\mathbf{k}_{3}$ the final neutrino momenta, $q=k_{\nu}-k_{3}$ the transferred energy-momentum, and $\chi=\cos \theta=\hat{\mathbf{k}}_{\nu} \cdot \hat{\mathbf{k}}_{3}$. The dynamical structure factors $\mathcal{S}^{(S)}(q, T)$ describe the response of nuclear matter to excitations induced by neutrinos, and they contain the relevant information on the medium. The vector (axial) part of the neutral current gives rise to density (spin-density) fluctuations, corresponding to the $S=0(S=1)$ spin channel. Density and spin-density responses con- 
tribute to the scattering of neutrinos, while the isospin-flip response contribute to neutrino absorption process, which is known to dominate the total cross section [10]. In the following, we shall show that large isoscalar fluctuations can give the dominant contribution at subnuclear densities.

Clearly, an enhancement of the response function in either channel will result in a quenching of the neutrino mean-free path. It has been shown [11] that at densities higher than $\rho_{0}$ a ferromagnetic instability can appear, leading consequently to a zero neutrino mean-free path [12]. However, the density at which such an instability could appear is not yet a settled question. For instance, whereas all Skyrme effective interactions predict a ferromagnetic transition for densities less than $\sim 3.5 \rho_{0}$ [13], recent microscopic calculations $[14,15]$ push it to higher densities, in case it exists.

At present, a large nuclear community is currently studying the liquid-gas phase transition which occurs during multi-fragmentation experiments in heavyion collisions. A present status is reviewed in the recent article of $\mathrm{Ph}$. Chomaz et al. [16] and we recall the main features directly related to our purpose. Such a transition appears below the saturation density $\rho_{0}$ of nuclear matter, at around $\sim 0.6 \rho_{0}$, i.e. $\sim 0.1 \mathrm{fm}^{-3}$. Heavy nuclei are located on the liquefaction curve for $T=0 \mathrm{MeV}$ and the critical temperature is located between 15 and 20 $\mathrm{MeV}$, depending on the specific nuclear model used. The onset of liquid-gas coexistence phase is associated to the spinodal instability, related to a negative curvature in the nucleonic free energy density such that the speed of sound vanishes. The analysis of the topology of the thermodynamic potential show that the phase transition is first order in symmetric and asymmetric nuclear matter. A unique spinodal instability is expected and the asymmetry do not modify its properties $[17,18]$. The spinodal instability is isoscalar (chemical instability), because the interaction between protons and neutrons is attractive at low density. In the density regime close to the spinodal, the density response function will be enhanced, thus affecting the un-charged current neutrino opacity while the charge current neutrino opacity is not modified. This may result in a reduced total neutrino mean-free path.

It is worth pointing out that Sawyer obtained a quite similar result, in his investigation on the dependence of the neutrino opacity and the equation of state of pure neutron matter [19]. Assuming that the neutrino scattering is dominated by the classical fluctuations, he concluded that a local softening of the equation of state could produce a thin layer, near to the star surface, quite opaque to neutrinos of some energy. In this work we consider asymmetric nuclear matter and we substantiate Sawyer's crude estimate by calculating consistently both the density fluctuations and the nuclear equation of state using the Gogny D1P finite-range nucleon-nucleon effective interaction [20]. We describe the nuclear isoscalar and isovector density fluctuations within the Hartree-Fock (HF) plus the Random Phase Approximation (RPA). The 

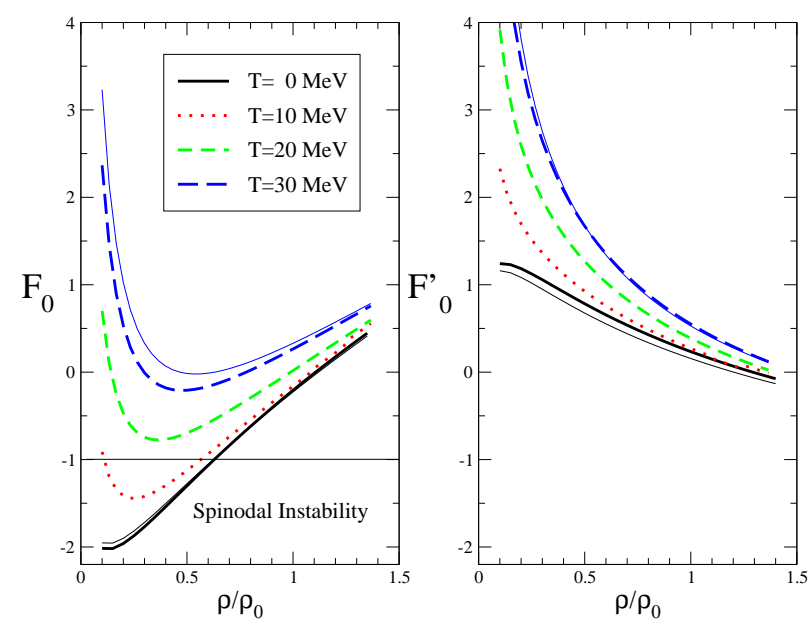

Fig. 1. (color online) The adimensional Landau parameters $F_{0}$ and $F_{0}^{\prime}$ are plotted as a function of the density at several values of temperature. The thin line represent a calculation of the same quantity in asymmetric nuclear matter with $x_{p}=0.25$ and for $T=0$ and $30 \mathrm{MeV}$. The definitions $F_{0}=\left(F_{0}^{n n}+F_{0}^{p p}+2 F_{0}^{n p}\right) / 2$ and $F_{0}^{\prime}=\left(F_{0}^{n n}+F_{0}^{p p}-2 F_{0}^{n p}\right) / 2$ has been taken and the coefficients $F_{0}^{\tau \tau \prime}$ are the second derivative of the free energy density with respect to the densities. The onset for the spinodal instability is given by $F_{0}=-1$.

calculation of the RPA response functions is performed in the Landau approximation, ignoring the Landau coefficients beyond the dipole one in both density and spin channels [21]. It is worth recalling that some values of Landau parameters are related to different instabilities. In particular, the density at which the dimensionless Landau parameter $F_{0}$ equals -1 indicates the onset of the spinodal instability. In Fig. 1, we show the Landau parameters $F_{0}$ and $F_{0}^{\prime}$ versus the total density for symmetric nuclear matter and several densities. The thin line correspond to asymmetric nuclear matter with $x_{p} \equiv Z /(N+Z)=0.25$. The results in asymmetric nuclear matter look very similar and we clearly see that out of the spinodal region, $F_{0}$ is attractive for a wide range of densities and temperatures. The Landau parameter $F_{0}^{\prime}$ which is responsible to isospin-flip fluctuations do not show any instable behaviour, which confirms that the instability is isoscalar.

In Fig. 2 we plot the pressures and particle fractions as a function of the density for asymmetric nuclear matter at a temperature of $T=10 \mathrm{MeV}$ and leptonic fraction $Y_{l}=0.285$, defined as $\left(\rho_{e}+\rho_{\nu}\right) / \rho$. In the low density region $\rho<\rho_{0}$, the total pressure is mainly supported by electrons, and the contribution of neutrinos is about $20 \%$ or less [22]. Hence, an increase of the neutrino pressure by a factor of 5 will increase by a factor of 2 the total pressure.

Let us assume the extreme hypothesis that, after a few hundred milliseconds, all the neutrinos contained inside the star are trapped inside the thin layer near the surface, and that the initial distribution of neutrino density is quasiuniform. Therefore, the increase of the neutrino pressure inside the thin layer 

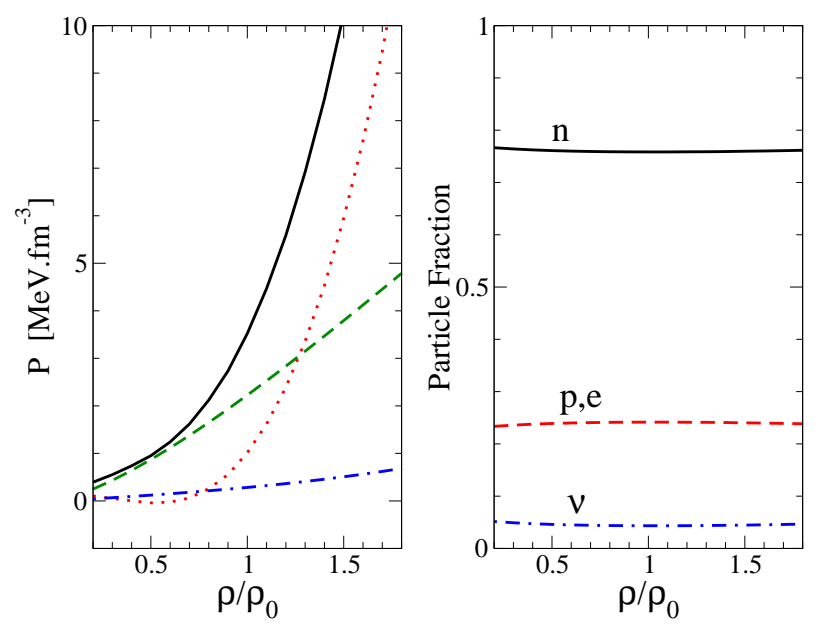

Fig. 2. (color online) Pressures (left panel) and particle fractions (right panel) plotted as a function of the density for asymmetric nuclear matter at temperature $\mathrm{T}=10 \mathrm{MeV}$ and leptonic fraction $Y_{l}=0.285$, calculated with the Gogny D1P effective interaction. The total pressure (solid line) is the sum of the nuclear (dotted line), the electronic (dashed line) and the neutrino (dot-dashed line) pressures. On the right panel, solid, dashed and dot-dashed lines refer to the particle fraction of neutrons, charged particles (i.e. protons and electrons), and neutrinos, respectively.

is given by the ratio of the PNS radius $R$ over the width $\epsilon$ of the thin layer and writes

$$
\frac{P_{\text {layer }}}{P_{\text {inside }}} \sim\left(\frac{R}{3 \epsilon}\right)^{4 / 3}
$$

where the neutrino pressure is polytropic versus the density with the power $4 / 3$. Hence, for a typical radius of $10 \mathrm{~km}$ and a width of $100 \mathrm{~m}$, the neutrino pressure increases by a factor greater than 200. Of course, the Pauli principle should strongly moderate this factor, but this schematic calculation points out the possible efficiency of the neutrino trapping.

This qualitative calculation assumes that all species are independent from each other. In fact, neutrinos are in $\beta$-equilibrium, so that a modification of their number implies a corresponding change of the number of other particles, in particular the electrons. If the neutrino density is increased at constant temperature and constant baryonic density, then the $\beta$ equilibrium shifts in the direction to raise the number of electrons and protons. This is the consequence of Le Chatelier's principle of minimum constraint extended to quantum systems. The same principle predicts that when the temperature is increased, the system tends to move towards the endothermic direction, thus increasing the number of electrons and protons. Consequently, raising both neutrino density and temperature produces electrons and protons. As electrons are mainly responsible for the pressure at low density, the overall effect of neutrino trapping is the increase of the total pressure. 

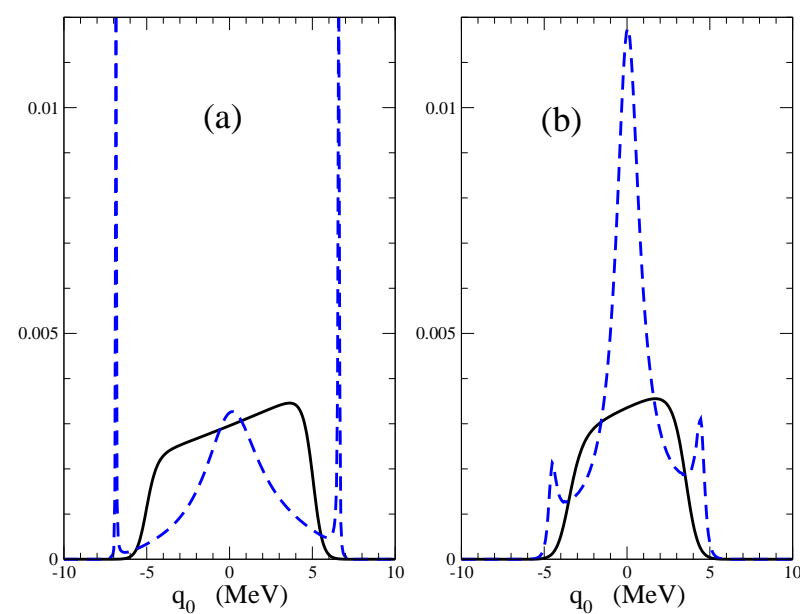

Fig. 3. (color online) The density-density response function $\mathcal{S}^{(0)}(q, T)$ is plotted as a function of the transfered energy for two values of the density: (a) $\rho_{0}$ and (b) $0.75 \rho_{0}$. The calculations have been done for momentum $q=10 \mathrm{MeV}$, temperature $T=10 \mathrm{MeV}$ and leptonic fraction $Y_{l}=0.285$. The solid and dashed lines stand for the HF and the RPA results respectively.

Now we concentrate on the mechanism responsible for neutrino trapping. In the following, we will approach the liquid-gas phase transition from the homogeneous nuclear matter. This can be done by decreasing either the density at constant temperature or the temperature at constant density. In both cases, we should keep the density beyond the spinodal one or the temperature beyond the critical one. The density-density response function $\mathcal{S}^{(0)}(q, T)$ is represented in Fig. 3 for density values approaching the spinodal point, namely $\rho_{0}$ (panel a) and $0.75 \rho_{0}$ (panel b). The solid line stands for the HF calculation while the dashed line includes the RPA correlations, with a particle-hole interaction described in terms of the monopolar and dipolar Landau parameters. In the left panel, we see two symmetric collective states inside the continuum strength. These states indicate that near the Fermi surface, the residual interaction is repulsive. It can be seen that when the system approaches the liquid-gas phase transition, a zero energy mode becomes increasingly enhanced. In fact, the peak diverges at the spinodal density. This is a signature of a strong attractive residual interaction, which indeed induces the phase transition.

The neutrino mean-free path as a function of the density is plotted in Fig. 4 for a temperature $T=10 \mathrm{MeV}$ and a leptonic fraction $Y_{l}=0.285$. Left and right panels correspond to $\mathrm{HF}$ and RPA results, respectively. One can see that the RPA correlations induce a strong reduction of the neutrino meanfree path inside the liquid-gas phase transition in agreement with the previous results of R. Sawyer [19]. Hence, neutrinos could be massively trapped inside the thin external layer with densities near the densities of the liquid-gas phase transition, and a radius close to the radius of the proto-neutron star. The spinodal density is close to $0.6 \rho_{0}$ in symmetric nuclear matter and is nearly constant in asymmetric nuclear matter until $\rho_{x} / \rho \sim 0.1(x=n$ or $p)$ [18]. 

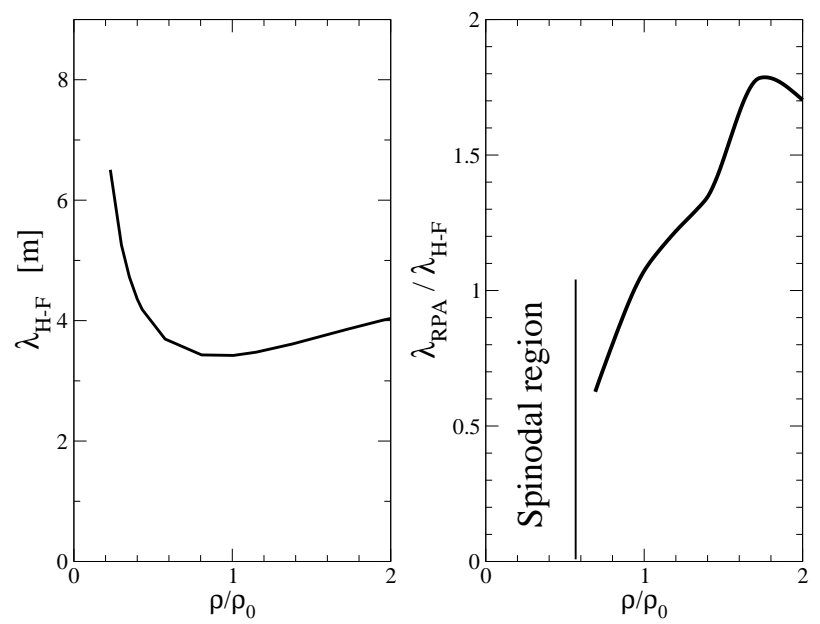

Fig. 4. The neutrino mean-free path is plotted as a function of the density for asymmetric nuclear matter at $T=10 \mathrm{MeV}$ and $Y_{l}=0.285$. Left pannel: HF result, right pannel: ratio between RPA and HF mean-free paths.

Of course, the RPA scheme can be questionable in such a large fluctuations regime. The result of effects as short-range correlations, possible contributions of two or more loop processes at these densities have to be elucidated, as well as the response to neutrino probes of inhomogeneous nuclear matter inside the phase coexistence. Nevertheless, we believe that our approach reflects the basic physics near the spinodal point.

Let us now analyze the physical consequences for the supernovae-II mechanism due to the interplay between neutrino propagation and the liquid-gas phase transition. During the iron core collapse, neutrino are trapped inside the core. The energy gained by the collapse is essentially carried away by the neutrinos after the bounce. Neutrinos are massively trapped for densities below the saturation one, and hence those neutrinos which are diffusing from the inner part of the PNS cannot reach the outer part of the star. An important fraction of neutrinos accumulates inside a thin layer close to the surface of the PNS. The neutrino Fermi energy increases and shifts the beta equilibrium to the production of protons and electrons. The neutrino and electronic pressures increase as well as the temperature inside the thin layer. We believe that this effect may lead to a partial conversion of the energy carried away by the neutrino gas to the matter through the beta equilibrium. According to the Euler transport equation, a local increase of pressure will produce an ejection of matter. This radiated matter will encounter the shock wave and may contribute to its revival. Indeed, the energy carried away by neutrinos represents about $98 \%$ of the collapse energy while the shock wave transports only $2 \%$ of it. If the ejection of particles carries away only $2 \%$ of neutrino energy, the total kinetic energy of matter will increased by a factor of 2 . This is the reason why we believe that this new scenario may contain an efficient mechanism for the explosion. 
This scenario may modify the neutrino emission signal from supernovae. Indeed, at the beginning of the collapse (for $\rho \sim 10^{10} \mathrm{~g} / \mathrm{cm}^{3}$ ), an electronic neutrino burst should come from the electronic capture. Then neutrinos are trapped and do not radiate from the PNS. After the bounce, if the proposed mechanism is strong enough to produce a second shock wave, the neutrinos "englued" inside the thin layer would be massively liberated. In this case, a second neutrino burst, mixing the leptonic flavors, would be observed. The observation of two neutrino bursts could be a signature of the second explosion. However, this scenario do not necessary imply a second shock wave but could produce only a huge ejection of matter. Then, we stress that the nonobservation of two neutrino bursts is not in contradiction with this scenario.

In this letter we have explored the consequences that large density fluctuations inside the liquid-gas phase transition could have for neutrino propagation and supernovae-II explosion. The calculations presented here are very schematic and apply only to the homogeneous phase close to the coexistence region, either for densities higher than the spinodal one, or temperatures higher than the critical one. For instance, close to the critical temperature, we expect to observe the coherent scattering of neutrinos in analogy with the well-known critical opalescence phenomenon (with respect to photons) observed in condensed matter. Recently, neutrino opacity has also been found to become very high inside the pasta phase, due to the coherent scattering of neutrinos off clusters [23]. This indicate that inhomogeneous nuclear matter should also trapp neutrinos, and increase the efficiency of our scenario. In conclusion, this article focuses on neutrino propagation close to the liquid-gas phase transition in nuclear matter and shows that not trivial medium effects can trapp neutrinos. Based on this effect, we propose to include the trapping in hydrodynamical simulations. Work in this direction is now in progress.

\section{Acknowledgments}

Interesting discussions with M. Baldo, Ph. Chomaz, H. Müther, A. PérezCañellas and A. Sedrakian are gratefully acknowledged. J.M. wants also to thank N. Van Giai and late D. Vautherin for introducing him into the subject of neutrino propagation in nuclear matter. J.N. is supported by MCyT/FEDER (Spain), grant number BMF2001-0262, and Generalitat Valenciana, grant number GV01-216.

\section{References}

[1] S.A. Colgate and R.H. White, Astrophys. J. 143 (1966) 626.

[2] W.D. Arnett, Can. J. Phys. 44 (1966) 2553. 
[3] R. Buras, M. Rampp, H.-Th. Janka, and K. Kifonidis, Phys. Rev. Lett. 90 (2003) 241101.

[4] H.A. Bethe and J.R. Wilson, Astrophys. J. 295 (1985) 14.

[5] P. Blottiau, Ph. D. thesis, Paris VII University (1989).

[6] S. Bruenn, Astrophys. J. Suppl. 58 (1985) 771.

[7] P. Mellor, J.P. Chièze and J.L. Basdevant, Astron. Astrophys. 197 (1988) 123.

[8] M. Rampp and H.T. Janka, Astron. Astrophys. 396 (2002) 361.

[9] N. Iwamoto and C.J. Pethick, Phys. Rev. D 25 (1982) 313.

[10] S. Reddy, M. Prakash and J.M. Lattimer, Phys. Rev D 58 (1998) 013009.

[11] A. Vidaurre, J. Navarro and J. Bernabeu, Astron. Astrophys. 135 (1984) 361.

[12] J. Navarro, E.S. Hernández and D. Vautherin, Phys. Rev. C 60 (1999) 045801.

[13] J. Margueron, J. Navarro and Nguyen Van Giai, Phys. Rev. C 66 (2002) 014303.

[14] S. Fantoni, A. Sarsa and K.E. Schmidt, Phys. Rev. Lett. 87 (2001) 181101.

[15] I. Vidaña and I. Bombaci, Phys. Rev. C 66 (2002) 045801.

[16] Ph. Chomaz et al., Phys. Rep. 389 (2004) 263.

[17] V. Baran et al., Phys. Rev. Lett. 86 (2001) 4492.

[18] J. Margueron and Ph. Chomaz, Phys. Rev. C 67 (2003) 041602(R).

[19] R. Sawyer, Phys. Rev. D 11 (1975) 2740.

[20] M. Farine et al., J. Phys. G: Nucl. Part. Phys. 25 (1999) 863.

[21] J. Margueron, Ph.D. Thesis, Paris XI University (2001).

[22] E. Suraud, Ph.D. thesis, Paris VI University (1984).

[23] C.J. Horowitz, M.A. Pérez-García and J. Piekarewicz, astro-ph/0401079. 